\title{
ON INCOMPLETE NUMERICAL FUNCTIONS
}

BY J. V. USPENSKY

1. Introduction. Numerical functions depending on divisors of an integer find important applications in various questions of higher arithmetic. In general they are defined by sums of the type

$$
\sum f(d, \delta)
$$

extended over conjugate divisors of a given integer, these being subject, sometimes, to certain limitations. If these limitations are expressed in the form of an inequality such as $d<\delta$ or $d<3 \delta$ we call the numerical function incomplete.

The first example of the use of such functions in an important arithmetical problem is due to Liouville. For an odd integer $m$ let $g(m)$ be defined as a sum

$$
g(m)=\sum(-1)^{(d-1) / 2}
$$

extended over divisors of $m$ which are less than their conjugate; in other words, the sum extends over all representations of $m$ in the form $m=d \delta$ where $d$ and $\delta$ are positive integers and $d<\delta$.

If, on the other hand,

$$
N\left(n=x^{2}+y^{2}+z^{2}\right)
$$

denotes, as usual, the number of representations of $n$ as a sum of three squares, then for $n \equiv 3(\bmod 8)$ we have this peculiar result:

$$
\begin{aligned}
N\left(n=x^{2}+y^{2}+z^{2}\right) & \\
=8\left[g(n)+2 g\left(n-4 \cdot 1^{2}\right)+\right. & 2 g\left(n-4 \cdot 2^{2}\right) \\
& \left.+2 g\left(n-4 \cdot 3^{2}\right)+\cdots\right] .
\end{aligned}
$$

In the right member, the series is continued so far as the arguments remain positive.

Aside from its obvious practical value this result of Liouville's is very curious in another respect; this may be discovered by comparing it with a different expression of the same number. 
Denoting by $\rho(m)$ a complete function

$$
\rho(m)=\sum(-1)^{(d-1) / 2},
$$

where the sum extends over all the divisors $d$ of an odd integer $m$, we have also

$$
\begin{aligned}
N\left(n=x^{2}\right. & \left.+y^{2}+z^{2}\right) \\
& =8\left[\rho\left(\frac{n-1^{2}}{2}\right)+\rho\left(\frac{n-3^{2}}{2}\right)+\rho\left(\frac{n-5^{2}}{2}\right)+\cdots\right] .
\end{aligned}
$$

In both cases the right hand members are built similarly, but while in the second expression the sum in the right hand member is suggested by the fact that $4 \rho(m)$ is a number of representations of $m$ as a sum of two squares, no similar origin can be devised for the right hand member of the first expression. At least it seems that the function $g(m)$ has nothing to do with the number of representations by binary forms.

Since the time when this first result by Liouville became known several such results have been discovered, mostly by the use of elliptic functions. It is the purpose of this note to show how all similar results can be derived in the most elementary manner from certain very general formulas which I have had in my possession for years, but hitherto had no opportunity to publish in full with their implications concerning incomplete numerical functions. However, it should be noted that in my paper $O n$ Gierster's class-number relations* important use has been made of formulas of this type.

2. The Fundamental Formulas. The general formulas we shall establish first contain a function $F(x, y, z)$ defined for integral values of its arguments and satisfying the following conditions of parity:

$$
F(-x, y, z)=F(x, y, z), F(x,-y,-z)=-F(x, y, z) .
$$

Moreover

$$
F(x, 0, z)=0
$$

whenever the middle argument $y$ can vanish. Aside from these requirements there are no other conditions imposed on $F(x, y, z)$.

* American Journal of Mathematics, vol. 50 (1928), No. 1. 
In the selection of this function we therefore possess a high degree of freedom.

Let $n$ be an arbitrary positive integer. We consider first the equation

$$
n=i^{2}+d \delta,
$$

where $i$ may have any integral value, while $d$ and $\delta$ are positive integers. The sum

$$
S=\sum_{(a)} F(d-2 i, \delta-d+i, i)
$$

is extended over all the solutions of the equation (a). On the other hand, consider the integral solutions of the equation

$$
4 n=h^{2}+\Delta \Delta^{\prime}
$$

where $\Delta$ and $\Delta^{\prime}$ are positive integers satisfying the conditions

$$
3 \Delta<\Delta^{\prime}, \quad \Delta^{\prime}+\Delta \equiv 0(\bmod 4) .
$$

Let us now form another sum

$$
\begin{aligned}
S^{\prime}= & \sum_{(b)}\left\{F\left(h, \frac{\Delta^{\prime}-3 \Delta}{4}, \frac{\Delta-h}{2}\right)\right. \\
& \left.\quad-F\left(\frac{\Delta^{\prime}+3 \Delta-2 h}{4}, \frac{\Delta^{\prime}-3 \Delta}{4}, \frac{\Delta-h}{2}\right)\right\} .
\end{aligned}
$$

There is a simple relation between the sums $S$ and $S^{\prime}$; namely,

$$
S=S^{\prime}
$$

when $n$ is not a perfect square; but if $n=s^{2}$,

$$
S=S^{\prime}+\sum_{j=1}^{s-1}\{F(2 s, s-j, s)-F(2 s-j, s-j, s)\} .
$$

This relation can be exhibited more explicitly as follows:

$$
\begin{aligned}
& \sum_{(a)} F(d-2 i, \delta-d+i, i) \\
= & \sum_{(b)}\left\{F\left(h, \frac{\Delta^{\prime}-3 \Delta}{4}, \frac{\Delta-h}{2}\right)\right. \\
& \left.-F\left(\frac{\Delta^{\prime}+3 \Delta-2 h}{4}, \frac{\Delta^{\prime}-3 \Delta}{4}, \frac{\Delta-h}{2}\right)\right\}+T
\end{aligned}
$$


where $T=0$, if $n$ is not a perfect square,

$$
T=\sum_{j=1}^{s-1}\{F(2 s, s-j, s)-F(2 s-j, s-j, s)\}, \text { if } n=s^{2} .
$$

This is our first general formula. The second is quite similar, but contains a function satisfying the conditions

$$
\begin{gathered}
F(-x, y, z)=-F(x, y, z), \quad F(x,-y,-z)=F(x, y, z), \\
F(x, 0, z)=0 .
\end{gathered}
$$

It can be written as follows:

$$
\begin{aligned}
& \sum_{(a)} F(d-2 i, \delta-d+i, i) \\
= & \sum_{(b)}\left\{F\left(h, \frac{\Delta^{\prime}-3 \Delta}{4}, \frac{\Delta-h}{2}\right)\right. \\
& \left.+F\left(\frac{\Delta^{\prime}+3 \Delta-2 h}{4}, \frac{\Delta^{\prime}-3 \Delta}{4}, \frac{\Delta-h}{2}\right)\right\}-T,
\end{aligned}
$$

where the extent of summations and the meaning of $T$ are the same as in (I).

Two more formulas of the same kind can be added. One of them is

$$
\begin{aligned}
& \sum_{(c)} F(\delta+i, \delta+i-d, i) \\
= & \sum_{(d)}\left\{F\left(\frac{\Delta^{\prime}+\Delta}{2}, \frac{\Delta^{\prime}-\Delta}{2}, \Delta-h\right)\right. \\
& \left.-F\left(h, \frac{\Delta^{\prime}-\Delta}{2}, \Delta-h\right)\right\}+T,
\end{aligned}
$$

for the function with the same conditions of parity as in (I), while for the function with the same parity as in (II) we have

$$
\begin{aligned}
& \sum_{(c)} F(\delta+i, \delta+i-d, i) \\
= & \sum_{(d)}\left\{F\left(\frac{\Delta^{\prime}+\Delta}{2}, \frac{\Delta^{\prime}-\Delta}{2}, \Delta-h\right)\right. \\
& \left.+F\left(h, \frac{\Delta^{\prime}-\Delta}{2}, \Delta-h\right)\right\}+T .
\end{aligned}
$$


In both cases the extent of the summations is as indicated below by (c) and (d) :

$$
\begin{aligned}
& n=i^{2}+2 d \delta, \\
& n=h^{2}+\Delta \Delta^{\prime}, \quad \Delta<\Delta^{\prime}, \quad \Delta^{\prime} \equiv \Delta(\bmod 2),
\end{aligned}
$$

and $T=0$ if $n$ is not a perfect square, but

$$
T=\sum_{j=0}^{s-1}\{F(j, j, s)-F(s, j, s)\}, \quad \text { if } \quad n=s^{2} .
$$

3. Proof of the Preceding Formulas. All these formulas were first discovered by what may be termed "groping in the dark," but, once discovered, their proof did not present any difficulty, and is, indeed, extremely simple. As the proof is the same in all cases, it suffices here to develop it in one case; for instance, in the case of the formula (I).

The sum $S$ can be split into three parts $S_{1}, S_{2}, S_{3}$ according as $\delta-d+i>0,<0$ or $=0$. The equations

$$
h=d-2 i, \Delta=d, \Delta^{\prime}=4 \delta-d+4 i
$$

establish a one-to-one correspondence between the solutions of

$$
n=i^{2}+d \delta
$$

with $\delta-d+i>0$ and the solutions of

$$
4 n=h^{2}+\Delta \Delta^{\prime}
$$

satisfying the conditions

(e) $0<3 \Delta<\Delta^{\prime}, \Delta^{\prime}+\Delta \equiv 0(\bmod 4), \Delta^{\prime}-\Delta+2 h>0$.

The second group of equations,

$$
h=\delta+2 i, \Delta=\delta, \Delta^{\prime}=4 d-\delta-4 i,
$$

establishes a one-to-one correspondence between the same solutions of (b) and the solutions of (a) with $\delta-d+i<0$. Thus

$$
\begin{aligned}
S_{1}+S_{2}= & \sum_{(e)}\left\{F\left(h, \frac{\Delta^{\prime}-3 \Delta}{4}, \frac{\Delta-h}{2}\right)\right. \\
& \left.+F\left(\frac{\Delta^{\prime}+3 \Delta-h}{4}, \frac{3 \Delta-\Delta^{\prime}}{4}, \frac{h-\Delta}{2}\right)\right\},
\end{aligned}
$$


or, because $F(x,-y,-z)=-F(x, y, z)$,

$$
\begin{aligned}
S_{1}+S_{2}= & \sum_{(e)}\left\{F\left(h, \frac{\Delta^{\prime}-3 \Delta}{4}, \frac{\Delta-h}{2}\right)\right. \\
& \left.-F\left(\frac{\Delta^{\prime}+3 \Delta-h}{4}, \frac{\Delta^{\prime}-3 \Delta}{4}, \frac{\Delta-h}{2}\right)\right\} .
\end{aligned}
$$

In the right member the sum is extended over solutions of (a) satisfying the conditions (e). If, on the other hand, $h, \Delta, \Delta^{\prime}$ run over the solutions of (b) satisfying the conditions

$$
0<3 \Delta<\Delta^{\prime}, \Delta^{\prime}+\Delta \equiv 0(\bmod 4), \Delta^{\prime}-\Delta+2 h<0,
$$

the numbers $h_{1}, \Delta_{1}, \Delta_{1}{ }^{\prime}$ defined by the equations

$$
h_{1}=-\frac{\Delta^{\prime}+3 \Delta-2 h}{4}, \Delta_{1}=\frac{\Delta-\Delta^{\prime}+2 h}{4}, \Delta_{1}^{\prime}=\frac{\Delta^{\prime}-\Delta-6 h}{4},
$$

reproduce exactly the same set. It is obvious therefore that

$$
\begin{aligned}
\sigma= & \sum_{(f)}\left\{F\left(h, \frac{\Delta^{\prime}-3 \Delta}{4}, \frac{\Delta-h}{2}\right)\right. \\
& \left.-F\left(\frac{\Delta^{\prime}+3 \Delta-2 h}{4}, \frac{\Delta^{\prime}-3 \Delta}{4}, \frac{\Delta-h}{2}\right)\right\} \\
= & \sum_{(f)}\left\{F\left(h_{1} \frac{\Delta_{1}^{\prime}-3 \Delta_{1}}{4}, \frac{\Delta_{1}-h_{1}}{2}\right)\right. \\
& \left.-F\left(\frac{\Delta_{1}^{\prime}+3 \Delta_{1}-2 h_{1}}{4}, \frac{\Delta_{1}^{\prime}-3 \Delta_{1}}{4}, \frac{\Delta_{1}-h_{1}}{2}\right)\right\} .
\end{aligned}
$$

But, by the properties of $F(x, y, z)$, we have

$$
\begin{aligned}
& F\left(h_{1}, \frac{\Delta_{1}^{\prime}-3 \Delta_{1}}{4}, \frac{\Delta_{1}-h_{1}}{2}\right) \\
& =F\left(\frac{\Delta^{\prime}+3 \Delta-2 h}{4}, \frac{\Delta^{\prime}-3 \Delta}{4}, \frac{\Delta-h}{2}\right), \\
& F\left(\frac{\Delta_{1}^{\prime}+3 \Delta_{1}-2 h_{1}}{4}, \frac{\Delta_{1}^{\prime}-3 \Delta_{1}}{4}, \frac{\Delta_{1}-h_{1}}{2}\right) \\
& =F\left(h, \frac{\Delta^{\prime}-3 \Delta}{4}, \frac{\Delta-h}{2}\right),
\end{aligned}
$$


so that the preceding equation gives

$$
\sigma=-\sigma
$$

whence $\sigma=0$. This important conclusion shows that

$$
\begin{aligned}
S_{1}+S_{2}= & \sum\left\{F\left(h, \frac{\Delta^{\prime}-3 \Delta}{4}, \frac{\Delta-h}{2}\right)\right. \\
& \left.-F\left(\frac{\Delta^{\prime}+3 \Delta-2 h}{4}, \frac{\Delta^{\prime}-3 \Delta}{4}, \frac{\Delta-h}{2}\right)\right\},
\end{aligned}
$$

where the sum extends over solutions of (b) subject to the conditions

$$
0<3 \Delta<\Delta^{\prime}, \Delta^{\prime}+\Delta \equiv 0(\bmod 4), \Delta^{\prime}-\Delta+2 h \neq 0 .
$$

But $\Delta^{\prime}-\Delta+2 h$ can be zero only if $n$ is a perfect square, say $s^{2}$, and the corresponding part of the sum is in this case

$$
-\sum_{j=0}^{s-1}\{F(2 s, s-j, s)-F(2 s-j, s-j, s)\} .
$$

On the other hand $S_{3}=0$ because of the property $F(x, 0, z)=0$. Both these remarks, combined with the above established nontrivial equation, lead to the formula (I).

4. Various Applications. By selecting the highly arbitrary function $F(x, y, z)$ in a proper way we can obtain a great many more or less interesting results implicitly contained in the above established formulas. Here we confine ourselves to a few consequences of our general formulas bearing upon the problems of representation of numbers by sums of three and five squares. The discussion leading to the final statements in all cases is so easy that without causing any difficulties to the reader we can omit all the details of reasoning.

(a) Let us suppose $n \equiv 3(\bmod 8)$ in (III). It is easy to see that the first argument in $F(x, y, z)$ is even throughout and the third argument is odd throughout. We are therefore entitled to take

$$
F(x, y, z)=(-1)^{x / 2+(z-1) / 2} .
$$

The resulting identity if properly interpreted leads to the following conclusion. 
If $N_{3}(n)$ is the number of representations of $n$ as a sum of three squares and

$$
g(m)=\sum(-1)^{(d-1) / 2}, \quad m=d d^{\prime}, \quad d<d^{\prime},
$$

then

$$
N_{3}(n)=8\left[g(n)+2 g\left(n-2^{2}\right)+2 g\left(n-4^{2}\right)+\cdots\right] .
$$

This is obviously Liouville's result, which was mentioned in the introduction.

(b) If, supposing $n \equiv 3(\bmod 4)$, we take

in (III) or

$$
F(x, y, z)=\cos \frac{\pi x}{4} \sin \frac{\pi z}{4}
$$

$$
F(x, y, z)=\sin \frac{\pi x}{4} \cos \frac{\pi z}{4}
$$

in (IV), introduce a new incomplete function

$$
G(m)=\sum(-1)^{(d-1) / 2+\left(d^{2}-1\right) / 8}, \quad m=d d^{\prime}, \quad d<d^{\prime},
$$

and set

$$
\begin{aligned}
& P=G(n)+2 G\left(n-4^{2}\right)+2 G\left(n-8^{2}\right)+\cdots, \\
& Q=2 G\left(n-2^{2}\right)+2 G\left(n-6^{2}\right)+2 G\left(n-10^{2}\right)+\cdots, \\
& R=g(n)-2 g\left(n-4^{2}\right)+2 g\left(n-8^{2}\right)-\cdots,
\end{aligned}
$$

then we obtain the following results:

$$
\begin{aligned}
& N_{3}(2 n)=24\left[P+(-1)^{(n-7) / 8} R\right] \text { if } n \equiv 7(\bmod 8), \\
& N_{3}(2 n)=24\left[Q+(-1)^{(n-3) / 8} R\right] \text { if } n \equiv 3(\bmod 8) .
\end{aligned}
$$

(c) If, for $n \equiv 5(\bmod 8)$, we define $F(x, y, z)$ in (III) as follows :

$$
\begin{aligned}
& F(x, y, z)=0, \text { if } x \text { is even } \\
& F(x, 0, z)=0, \\
& F(x, y, z)=\cos \frac{\pi x}{4} \sin \frac{\pi z}{4}, \text { otherwise, }
\end{aligned}
$$

the result obtained is rather curious; namely, setting

$$
\begin{aligned}
& S=g\left(\frac{n-1^{2}}{4}\right)+g\left(\frac{n-3^{2}}{4}\right)+g\left(\frac{n-5^{2}}{4}\right)+\cdots, \\
& T=G(n)-2 G\left(n-4^{2}\right)+2 G\left(n-8^{2}\right)-\cdots,
\end{aligned}
$$


we have

$$
N_{3}(n)=12\left[2 S+(-1)^{(n-5) / 8} T\right]+12 \sum(-1)^{(j-1) / 2},
$$

where the last sum is extended over all the solutions of the equation

$$
n=i^{2}+4 j^{2}
$$

in positive numbers.

(d) If, for $n \equiv 1(\bmod 8)$, we suppose in (III) and (IV) that $F(x, y, z)$ vanishes whenever $y$ is even, but otherwise is respectively

and

$$
F(x, y, z)=\cos \frac{\pi x}{4} \sin \frac{\pi z}{4}
$$

$$
F(x, y, z)=\sin \frac{\pi x}{4} \cos \frac{\pi z}{4},
$$

and take the difference of the results thus obtained, the conclusion is as follows. Using the notation

$$
\begin{aligned}
h(m) & =\sum(-1)^{(d-1) / 2}, m=d d^{\prime}, d<d^{\prime}, d \text { odd } \\
h(0) & =0, \\
H(m) & =\sum\left(-2 /\left(d^{\prime}-d\right)\right), m=d d^{\prime}, d<d^{\prime}, d \text { odd }, \\
H(0) & =\frac{1}{2},
\end{aligned}
$$

and

$$
\begin{aligned}
& L=H\left(\frac{n-1^{2}}{4}\right)-H\left(\frac{n-3^{2}}{4}\right)-H\left(\frac{n-5^{2}}{4}\right) \\
&+H\left(\frac{n-7^{2}}{4}\right)+H\left(\frac{n-9^{2}}{4}\right)-\cdots, \\
& M=h\left(\frac{n-1^{2}}{4}\right)+h\left(\frac{n-3^{2}}{4}\right)+h\left(\frac{n-5^{2}}{4}\right)+\cdots,
\end{aligned}
$$

we have

$$
N_{3}(n)=12(L+2 M) .
$$

(e) A curious result can be obtained from (III) if, supposing $n \equiv 3(\bmod 8)$, we take

$$
F(x, y, z)=(-1)^{x / 2} z .
$$


Denoting by $\omega(m)$ the sum of divisors which are less than their conjugate, we obtain

$$
\omega(n)-2 \omega\left(n-2^{2}\right)+2 \omega\left(n-4^{2}\right)-\cdots=\sum(-1)^{(i-1) / 2} i,
$$

where the sum is extended over all the solutions of the equation

$$
n=i^{2}+j^{2}+k^{2}
$$

in positive odd numbers. A number of similar results can be derived from the same formulas (III) and (IV), but it would be too long to dwell upon them and we prefer to give some applications of (I) and (II).

5. Applications of Formulas (I) and (II).

(a) Suppose that in (I) the function $F(x, y, z)$ vanishes whenever $y$ is even, but otherwise

$$
F(x, y, z)=\cos \frac{\pi x}{4} \sin \frac{\pi z}{4} .
$$

Moreover, we assume $n \equiv 1(\bmod 4)$. If we introduce three incomplete functions

$$
\begin{aligned}
& \phi(m)=\sum(-1)^{(d-1) / 2}, m=d d^{\prime}, 3 d<d^{\prime} \\
& \Phi(m)=\sum(-1)^{(d-1) / 2+\left(d^{2}-1\right) / 8}, m=d d^{\prime}, 3 d<d^{\prime} \\
& \psi(m)=\sum(-1)^{d}\left(\frac{-2}{d^{\prime}+3 d}\right), m=d d^{\prime}, 3 d<d^{\prime}, d+d^{\prime} \text { odd } \\
& \psi(0)=\frac{1}{2}
\end{aligned}
$$

and set

$$
\begin{gathered}
A=\phi(n)+2 \phi\left(n-2^{2}\right)+2 \phi\left(n-4^{2}\right)+\cdots, \\
B=\Phi(n)-2 \Phi\left(n-4^{2}\right)+2 \Phi\left(n-8^{2}\right)-\cdots, \\
C=\psi\left(\frac{n-1^{2}}{4}\right)-\psi\left(\frac{n-3^{2}}{4}\right)-\psi\left(\frac{n-5^{2}}{4}\right)+\psi\left(\frac{n-7^{2}}{4}\right) \\
+\psi\left(\frac{n-9^{2}}{4}\right)-\cdots,
\end{gathered}
$$

we obtain

$$
\begin{aligned}
& N_{3}(n)=12\left[A+(-1)^{(n-5) / 8} B\right] \text { if } n \equiv 5(\bmod 8), \\
& N_{3}(n)=12[A+C] \text { if } n \equiv 1(\bmod 8)
\end{aligned}
$$


There exists an analogous formula for $n \equiv 3(\bmod 8)$.

(b) Suppose that, in the formula (II), the function is defined as follows :

$$
\begin{aligned}
& F(x, y, z)=0, \text { whenever } y \text { is even, } \\
& F(x, y, z)=0, \text { whenever } x \text { or } x / 2 \text { is odd, } \\
& F(x, y, z)=x, \text { otherwise, }
\end{aligned}
$$

and again $n \equiv 1(\bmod 4)$. If $n \equiv 5(\bmod 8)$ and $\chi(m)$ is an incomplete function defined by

$$
\chi(m)=\sum\left(d^{\prime}+3 d\right), m=d d^{\prime}, 3 d<d^{\prime},
$$

then

$$
N_{5}(n)=14\left(\chi(n)+2 \chi\left(n-2^{2}\right)+2 \chi\left(n-4^{2}\right)+\cdots\right),
$$

where $N_{5}(n)$ denotes the number of representations of $n$ as the sum of five squares. This noteworthy result is due to Hermite.

In the case $n \equiv 1(\bmod 8)$, we make use of the incomplete functions

$$
\begin{aligned}
& X(m)=\chi(m)-\chi\left(\frac{m}{4}\right), m \text { even, } \\
& \omega(m)=\sum\left(\frac{-1}{d^{\prime}+3 d}\right), m=d d^{\prime}, 3 d<d^{\prime}, d+d^{\prime} \text { odd. }
\end{aligned}
$$

If then we set

$$
\begin{aligned}
& F=X\left(\frac{n-1^{2}}{4}\right)+X\left(\frac{n-3^{2}}{4}\right)+X\left(\frac{n-5^{2}}{4}\right)+\cdots, \\
& G=\omega\left(\frac{n-1^{2}}{4}\right)-3 \omega\left(\frac{n-3^{2}}{4}\right)+5 \omega\left(\frac{n-5^{2}}{4}\right)-\cdots,
\end{aligned}
$$

extending these series so far as the arguments remain positive, we obtain as a final result

$$
N_{5}(n)=60[F-G]+10 V,
$$

where $V=0$, if $n$ is not a square,

$$
V=\frac{1}{4}\left(3 s-(-1)^{(s-1) / 2}\right)^{2} \quad \text { if } \quad n=s^{2} .
$$

Analogous results exist in the case $n \equiv 3(\bmod 4)$.

The preceding formulas are not mere curiosities, but have 
real practical value. To show this let us determine the number of representations of $n=1089=33^{2}$ as the sum of five squares. The necessary numbers are given in the table below.

\begin{tabular}{c|l|r|r}
\hline Arguments & \multicolumn{1}{|c|}{$d^{\prime}+3 d$} & $X(m)$ & $\omega(m)$ \\
\cline { 2 - 3 } 272 & 275 & 275 & -1 \\
270 & $273,99,57,141,63$ & 702 & 2 \\
266 & $269,59,139$ & 467 & -1 \\
260 & $263,67,77$ & 407 & -1 \\
252 & $255,93,57,55,75$ & 535 & -1 \\
242 & 245,127 & 372 & 0 \\
230 & $233,61,121$ & 415 & 3 \\
216 & $219,81,51$ & 351 & -1 \\
200 & $203,55,49$ & 307 & -1 \\
182 & $185,47,97$ & 329 & 1 \\
162 & $165,63,87,45$ & 360 & 0 \\
140 & $143,43,47$ & 233 & -3 \\
116 & 119,41 & 160 & 0 \\
90 & $93,39,33,51$ & 216 & 0 \\
62 & 65,37 & 102 & 2 \\
32 & 35 & 35 & -1 \\
\hline
\end{tabular}

From this table we easily find

$$
F=5266, G=162,60(F-G)=306240 ;
$$

and as in this case $n$ is a perfect square $\left(=33^{2}\right)$, we have

$$
V=49^{2}=2401, \quad 10 \mathrm{~V}=24010 .
$$

The number of representations of 1089 as the sum of five squares is therefore

$$
N_{5}(1089)=330250 .
$$

STANFORD UNIVERSITY 